\title{
Optimized Orbitrap HCD for Quantitative Analysis of Phosphopeptides
}

\author{
Yi Zhang, ${ }^{*}$ Scott B. Ficarro, ${ }^{*}$ Shaojuan Li, and Jarrod A. Marto \\ Department of Cancer Biology and Blais Proteomics Center, Dana-Farber Cancer Institute, Department of \\ Biological Chemistry and Molecular Pharmacology, Harvard Medical School, Boston, Massachusetts, USA
}

Despite the tremendous commercial success of radio frequency quadrupole ion traps for bottom-up proteomics studies, there is growing evidence that peptides decorated with labile post-translational modifications are less amenable to low-energy, resonate excitation MS/MS analysis. Moreover, multiplexed stable isotope reagents designed for MS/MSbased quantification of peptides rely on accurate and robust detection of low-mass fragments for all precursors. Collectively these observations suggest that beam-type or tandem in-space MS/MS measurements, such as that available on traditional triple quadrupole mass spectrometers, may provide beneficial figures of merit for quantitative proteomics analyses. The recent introduction of a multipole collision cell adjacent to an Orbitrap mass analyzer provides for higher energy collisionally activated dissociation (HCD) with efficient capture of fragment ions over a wide mass range. Here we describe optimization of various instrument and post-acquisition parameters that collectively provide for quantification of iTRAQ-labeled phosphorylated peptides isolated from complex cell lysates. Peptides spanning a concentration dynamic range of 100:1 are readily quantified. Our results indicate that appropriate parameterization of collision energy as a function of precursor $\mathrm{m} / \mathrm{z}$ and $z$ provides for optimal performance in terms of peptide identification and relative quantification by iTRAQ. Using this approach, we readily identify activated signaling pathways downstream of oncogenic mutants of Flt-3 kinase in a model system of human myeloid leukemia. (J Am Soc Mass Spectrom 2009, 20, 1425-1434) @ 2009 American Society for Mass Spectrometry

A lthough some of the earliest demonstrations of low-energy MS/MS for peptide sequence analysis were performed on triple quadrupole mass spectrometers [1], subsequent technical developments in quadrupole ion trap instrumentation, including improved scan functionality $[2,3]$, injection of externally-formed ions [4-6], extended mass range capability [7], use of helium buffer gas [3], and automated control of trapped ion populations [8], all combined to catapult trap-based instruments to the forefront of proteomics research. Concomitant advances in CPU and embedded systems provided for rapid and automated control of instrument parameters and yielded heretofore unattainable throughput for peptide sequence analysis via LC-MS/MS. Today, several years past the emergence of proteomics as a field of active research [9], it is clear that "typical" tryptic peptides are very amenable to sequence analysis via low-energy MS/MS. More recently, the introduction of hybrid geometries [10-12] and other instrument configurations [13, 14], along with protocols for enrichment of post-translationally modified peptides [15, 16], have dramatically increased the experimental dynamic range of

Address reprint requests to Dr. J. A. Marto, Department of Cancer Biology, Dana-Farber Cancer Institute, 44 Binney Street, Smith 1158A, Boston, MA 02115-6084, USA. E-mail: jarrod_marto@dfci.harvard.edu

* These authors contributed equally to this work. proteomics analyses. Collectively, these results have revealed that peptides decorated with labile modifications often are not well behaved under low-energy MS/MS conditions. For example, facile loss of phosphoric acid from the side chains of serine- and threoninephosphorylated peptides often limits the available sequence-specific information contained in associated MS/MS spectra [17, 18]. Interestingly, recent reports $[19,20]$ suggest that higher energy, or "triple quadrupole like," MS/MS can provide improved sequence analysis, particularly for phosphorylated peptides. In the work reported herein, we characterize various performance metrics of the higher energy dissociation mode (HCD) recently implemented on the Orbitrap instrument [21]. The use of an rf-only multipole collision cell also facilitates analysis of iTRAQ labeled peptides [22]. We leverage these analytical features to quantify phosphorylation cascades associated with constitutively active forms of the FLT-3 receptor tyrosine kinase in a model system of human myeloid leukemia.

\section{Materials and Methods}

All chemicals and reagents were of HPLC grade, purchased from Sigma-Aldrich (St. Louis, MO), and used without further purification. 
Table 1. The number of validated MS/MS spectra, measured and expected ratios of proteins in standard mixture. All ratios were calculated against intensity of iTRAQ reporter ion 114, unless otherwise indicated

\begin{tabular}{lcccc}
\hline \multicolumn{1}{c}{ Protein name } & Number of $\mathrm{MS}^{2}$ spectra & Measured ratio ( \pm relative standard deviation) & Expected \\
\hline \hline Bovine serum albumin & 36 & $1: 3.71( \pm 43 \%): 12.73( \pm 41 \%): 85.90( \pm 40 \%)$ & $1: 5: 20: 100$ \\
& & $0.014( \pm 57 \%): 0.046( \pm 41 \%): 0.15( \pm 18 \%): 1^{*}$ & $0.01: 0.05: 0.2: 1 *$ \\
Glycogen phosphorylase & 26 & $1: 0.17( \pm 17 \%): 0.071( \pm 26 \%): 0.030( \pm 54 \%)$ & $1: 0.2: 0.05: 0.02$ \\
& & $39.44( \pm 33 \%): 6.48( \pm 29 \%): 2.72( \pm 36 \%): 1^{*}$ & $50: 10: 2.5: 1^{*}$ \\
$\beta$-Lactoglobulin & 95 & $1: 2.03( \pm 12 \%): 2.13( \pm 16 \%): 1.23( \pm 19 \%)$ & $1: 2: 2: 1$ \\
Cytochrome $c$ & 62 & $1: 3.32( \pm 15 \%): 3.31( \pm 20 \%): 1.60( \pm 26 \%)$ & $1: 3: 3: 2$ \\
$\beta$-Casein & 19 & $1: 1.98( \pm 10 \%): 1.97( \pm 12 \%): 4.67( \pm 14 \%)$ & $1: 2: 2: 4$ \\
\hline
\end{tabular}

* Ratios were calculated against intensity of iTRAQ reporter ion 117.

\section{HCD Analysis of Standard Protein Digest with Known iTRAQ Ratios}

Standard proteins (BSA, cytochrome $c, \beta$-casein, $\beta$ lactoglobulin, glycogen phosphorylase) were digested with trypsin. Next, four samples were generated by mixing varying quantities of tryptic peptides from each protein digest, in the specific ratios shown in Table 1. Each of these mixtures was labeled with an iTRAQ isobaric reagent (114, 115, 116, and 117, respectively). After mixing, small aliquots, corresponding to $\sim 10-100$ fmol, were analyzed by LC-MS/MS on an Orbitrap-XL equipped with HCD collision cell. Precursors were selected in the linear trap with an isolation window of 2.6 Da, a target value of $2 \times 10^{5}$, and a maximum ion injection time of $250 \mathrm{~ms}$. Precursor peptides were then transferred to the HCD cell and subjected to fragmentation with a $45 \%$ normalized collision energy. Resulting fragment ions were detected in the Orbitrap mass analyzer at a resolution of 7500 (FWHM @ m/z=400). MS/MS spectra were extracted and searched using MASCOT. Individual scans were discarded if the $\mathrm{m} / \mathrm{z}$ region within the isolation window contained other precursors. Next, iTRAQ reporter ion intensities were extracted from the remaining MS/MS scans, corrected for isotope impurity, and their respective ratios plotted (Supplementary Figure S1, which can be found in the electronic version of this article).

\section{CE Energy Profile Analysis Based on Tryptic Peptides from Whole Cell Lysate}

Human K562 cells were cultured in RPMI 1640 media supplemented with $10 \%$ fetal bovine serum and $1 \%$ penicillin/streptomycin. Cells were grown at $37^{\circ} \mathrm{C}$ in $5 \% \mathrm{CO}_{2}$ and aliquots of $\sim 5 \mathrm{E} 7$ cells were harvested by centrifugation during log phase. Cells were washed twice with $20 \mathrm{~mL}$ phosphate buffered saline, and the resulting cell pellet was lysed with $3 \mathrm{~mL}$ of $8 \mathrm{M}$ urea, $100 \mathrm{mM}$ ammonium bicarbonate, and $30 \mu \mathrm{L}$ each of Sigma-Aldrich phosphatase inhibitor cocktails I and II. Protein concentration was determined using the Bradford Assay (Bio-Rad Laboratories, Hercules, CA). Proteins were reduced by adding dithiothreitol (DTT) to a final concentration of $10 \mathrm{mM}$ and incubat- ing for $30 \mathrm{~min}$ at $60^{\circ} \mathrm{C}$. Afterwards, iodoacetamide was added to a final concentration of $20 \mathrm{mM}$ and the solution was incubated for $30 \mathrm{~min}$ at room temperature in the dark. Proteins were diluted to a final volume of $12 \mathrm{~mL}$ in $0.1 \mathrm{M}$ ammonium bicarbonate and digested with trypsin $(150 \mu \mathrm{g})$ at $37^{\circ} \mathrm{C}$ overnight with end-over-end rotation. The resulting peptide solution was acidified with 10\% TFA, and desalted on a $\mathrm{C}_{18}$ solid-phase extraction cartridge.

Murine BaF3 cells were similarly cultured and processed. Desalted peptides (400 $\mu \mathrm{g}$ total) were labeled with iTRAQ reagents according to the manufacturer's instructions. Briefly, four $100 \mu \mathrm{g}$ aliquots of dried peptides were reconstituted with $70 \mu \mathrm{L}$ ethanol, $30 \mu \mathrm{L} 0.5 \mathrm{M}$ triethylammonium bicarbonate. One tube of iTRAQ reagent $(114,115,116$, and 117) was added to each solution, and the reaction was allowed to proceed for $1 \mathrm{~h}$ at room temperature. Derivatized peptides were combined, dried by vacuum centrifugation, and desalted on a $\mathrm{C}_{18}$ solidphase extraction cartridge.

Aliquots of tryptic peptides $(\sim 200 \mathrm{ng}$, unlabeled and iTRAQ labeled) were analyzed by automated nanoLC/ESI/MS using a Waters NanoAcquity UPLC system (Waters, Milford, MA) operated in a vented column configuration. Peptides were loaded on a precolumn $(100 \mu \mathrm{m} \times 4 \mathrm{~cm}$ POROS 10R2) at a flow rate of $4 \mu \mathrm{L} / \mathrm{min}$. After sample loading, peptides were eluted to an analytical column $(50 \mu \mathrm{m} \times 12 \mathrm{~cm}$ $3 \mu \mathrm{m}$ C18) using an acetonitrile gradient $(2 \%-30 \% \mathrm{~B}$ in $59 \mathrm{~min} ; \mathrm{A}=0.1 \%$ formic acid in water, $\mathrm{B}=0.1 \%$ formic acid in acetonitrile) at a flow rate of $\sim 100$ $\mathrm{nL} / \mathrm{min}$. Electrospray was performed using a spray voltage of $2.2 \mathrm{kV}$. The mass spectrometer (LTQ Orbitrap XL; ThermoFisher Scientific, San Jose, CA) was operated in data-dependent mode such that the four most abundant precursors were subjected to CAD (threshold $=1 \mathrm{E} 6$, isolation width $=3 \mathrm{Da}$, normalized collision energy $=35 \%$, activation $\mathrm{q}=0.25$, activation time $=30 \mathrm{~ms}$ ), and HCD (threshold $=1 \mathrm{E} 6$, isolation width $=3$ Da, activation time $=30 \mathrm{~ms}, \mathrm{~m} / \mathrm{z} 100-2000$ ). The HCD collision energy was varied from $25 \%$ to $50 \%$, and from $30 \%$ to $55 \%$ (both in increments of $2.5 \%)$, for unlabeled and labeled peptides, respectively. One analysis was done for each HCD collision 
energy value. Dynamic exclusion was implemented with a repeat count of 1 , and an exclusion duration of 20 s. Peak lists from CAD and HCD scans were generated using customized in-house software. HCD spectra, with and without de-isotoping, were matched to peptide sequences from a forward or reversed human protein database using MASCOT (Matrix Science, Inc., London, UK) with a precursor tolerance of $30 \mathrm{ppm}$, and a fragment ion tolerance of $0.02 \mathrm{Da}$. Search parameters included carbamidomethylation of cysteine as a fixed modification $(+57 \mathrm{Da})$, and oxidation of methionine residues $(+16$ Da) as a variable modification.

Quantitative Phosphoproteomics Analysis of Acute Myeloid Leukemia (AML) Model System (WT, $W T+F L, I T D, D 835 Y)$

Murine BaF3 cells that stably express wild-type (WT), constitutively active mutant forms (ITD and D835Y, respectively) of the receptor tyrosine kinase FLT-3 were grown in standard culture medium, with IL-3 added to support growth of WT cultures. To drive phosphorylation downstream of the WT kinase, cultures containing of WT FLT-3 were also treated with $50 \mathrm{ng} / \mathrm{mL}$ of FLT-3 ligand (FL) for $5 \mathrm{~min}$ before harvest. Cells were collected, rinsed with cold PBS, and lysed in $8 \mathrm{M}$ urea. Proteins were digested with trypsin after reduction with $10 \mathrm{mM}$ DTT and alkylation with $55 \mathrm{mM}$ iodoacetamide. For each biological condition (WT, WT + FL, ITD, D835Y), $100 \mu \mathrm{g}$ of tryptic peptides from $\sim 2 \times 10^{6}$ cells were labeled with isobaric iTRAQ reagents. Labeled peptides were mixed, and further fractionated offline on a $\mathrm{C}_{18}$ column $(530 \mu \mathrm{m} \times 20 \mathrm{~cm}$, POROS 10R2). Fractions (gradient $0 \% \mathrm{~B}-40 \% \mathrm{~B}$ in $60 \mathrm{~min}, \mathrm{~B}: 70 \%$ $\mathrm{MeCN} / 0.1 \%$ TFA) were collected every minute, "round-robin" style, into five tubes. Each fraction was lyophilized in a centrifugal speed-vac concentrator. Samples were stored at $-80^{\circ} \mathrm{C}$ until LC-MS analysis.

Each lyophilized fraction was reconstituted in $100 \mu \mathrm{L}$ of loading buffer (60\% MeCN, $1 \%$ TFA, 30\% lactic acid), and passed through a self-packed MassPREP (Waters, Milford, MA) capillary column $(530 \mu \mathrm{m} \times 10 \mathrm{~cm})$ at 4 $\mu \mathrm{L} / \mathrm{min}$. The metal oxide column was then rinsed with $200 \mu \mathrm{L}$ of loading buffer, $200 \mu \mathrm{L}$ of rinse buffer $(80 \%$ $\mathrm{MeCN}, 1 \% \mathrm{TFA}$ ), and finally $200 \mu \mathrm{L}$ of $0.1 \%$ TFA at $20 \mu \mathrm{L} / \mathrm{min}$. Enriched phosphopeptides were directly eluted onto a $\mathrm{C}_{18}$ precolumn $(100 \mu \mathrm{m} \times 4 \mathrm{~cm}$, POROS 10R2) at $2 \mu \mathrm{L} / \mathrm{min}$ using $75 \mu \mathrm{L}$ of $100 \mathrm{mM}\left(\mathrm{NH}_{4}\right)_{2} \mathrm{HPO}_{4}$, $5 \mathrm{mM}$ EDTA. The precolumn was then rinsed with $0.2 \mathrm{M}$ acetic acid (HPLC solvent A) for $10 \mathrm{~min}$ before connecting to an analytical column $\left(25 \mu \mathrm{m} \times 10 \mathrm{~cm}, 3 \mu \mathrm{m}\right.$ dia. $\mathrm{C}_{18}$ resin). Peptides were eluted with a $2 \mathrm{~h}$ LC gradient, and introduced via electrospray ionization into an OrbitrapXL. MS spectra were acquired with a target value of $2 \times$ $10^{6}$, maximum ion injection time of $500 \mathrm{~ms}$, and a mass resolution of 15,000 (FWHM @ $m / z=400)$. A minimum threshold of $1 \times 10^{5}$ was used for MS/MS, with a charge state range of $2^{+}$to $4^{+}$. Up to eight precursors could be selected for each cycle, with a 20 s exclusion time for each precursor selected for MS/MS. One linear trap CAD and one HCD MS/MS spectrum was acquired in series for each precursor (Figure 3a). An optimized collision energy (see the Results and Discussion section) was used for HCD scans. The data files were processed with in-house software to extract CAD and HCD scans into separate files for subsequent MASCOT searches. In addition, iTRAQ reporter ion peak intensity values were extracted from HCD scans, and then inserted in the header for corresponding CAD and HCD scans of the same precursor; this provided for convenient retrieval of both peptide sequence identification and reporter ion quantification data from the MASCOT search results. The two files were searched by MASCOT against a forward and reversed mouse protein database with the following parameters: the mass tolerance values were set to $30 \mathrm{ppm}$ for each precursor, 0.6 Da for LTQ-CAD scans and 0.02 Da for HCD scans. Other common parameters included fixed modifications on cysteine (carbamidomethyl), N-terminus (iTRAQ4plex) and lysine (iTRAQ4plex), and variable modifications on methionine and tyrosine (oxidation), and serine/threonine (phosphorylation). Scans with MASCOT score above 25 (FDR $<1 \%$ ) were extracted for further analysis. Relative quantification for these peptides was based on ITRAQ reporter ion peak intensities. For peptides with multiple MS/MS scans, the associated iTRAQ reporter signal intensity values were summed before calculation of reported ratios.

Kyoto Encyclopedia of Genes and Genomes (KEGG) pathway annotations were downloaded from the KEGG ftp resource. GenBank protein gi numbers from the peptide database search results were converted to entrez gene names and assigned to one or more KEGG pathways. For each pathway assigned to at least one gene, the following metrics were calculated:

1. $\mathrm{m}=$ Number of genes in search results with Kegg pathway annotation A.

2. $\mathrm{M}=$ Total number of genes in search result.

3. $n=$ Number of genes in protein database with Kegg pathway annotation A.

4. $N=$ Total number of genes in protein database.

The $P$-value of finding $\mathrm{m}$ genes in the protein list with pathway annotation A was calculated using Fisher's exact test. The probability that exactly $\mathrm{m}$ genes are annotated as pathway $\mathrm{A}$ is given by the probability mass function (pmf) of a hypergeometric distribution, where

$$
p m f=P(k=m)=\frac{\left(\begin{array}{l}
n \\
m
\end{array}\right)\left(\begin{array}{l}
N-n \\
M-m
\end{array}\right)}{\left(\begin{array}{l}
N \\
M
\end{array}\right)}
$$

The one-tailed $P$ value representing enrichment of pathway annotation $\mathrm{A}$ is given by: 


$$
p=\text { value }=\sum_{k=m}^{\min (M, n)} P(k)
$$

\section{Results and Discussion}

\section{HCD Provides for Relative Quantification of iTRAQ Labeled Peptides}

Recently, Olsen et al. [21] demonstrated that higher energy dissociation of peptides could be obtained in a rf-only, multipole collision cell located adjacent to the c-trap on an Orbitrap-XL instrument. Contrary to CAD in a quadrupole ion trap, low mass fragment ions $(\mathrm{m} / \mathrm{z}<100 \mathrm{Da})$ could be efficiently generated and detected, regardless of the precursor $\mathrm{m} / \mathrm{z}$ value. To evaluate the efficiency and performance of HCD specifically for iTRAQ labeled peptides, we prepared an iTRAQ labeled sample consisting of tryptic peptides derived from five standard proteins, with ratios spanning a range of 1:1 to 1:100 (see the Materials and Methods section). Consistent with previous reports [22], we observed $\sim 95 \%$ labeling efficiency, based on comparison of extracted ion chromatograms for fully- and partially-labeled peptide pairs (data not shown). Peptide ratios from manually validated HCD scans were calculated based on their corresponding iTRAQ reporter ion intensities, and plotted for each protein (Supplementary Figure S1). In the case of modest ratios, for which both reporter ion channels have reasonable signal intensities $(\mathrm{s} / \mathrm{n}>5: 1)$, the measured values are in good agreement with those in Table 1. However, for more extreme ratios, BSA and glycogen phosphorylase in Supplementary Figure S1, the standard deviation increased significantly. In these cases one iTRAQ channel, 114 and 117 for BSA and glycogen phosphorylase, respectively, exhibited low signal-to-noise and contributed proportionately larger error. For example, calculation of peptide ratios based on 114 (BSA) and 117 (glycogen phosphorylase) yielded relative standard deviations of $\sim 40 \%$ and $\sim 30 \%$, respectively, for these two proteins across all iTRAQ channels (Table 1, rows 1 and 2). In contrast, the use of 117 (BSA) and 114 (glycogen phosphorylase) to calculate peptide ratios resulted in a systematic increase in relative standard deviation for both proteins, the maximum of which corresponded with inclusion of the lowest intensity iTRAQ reporter. Collectively these data and observations demonstrate that iTRAQ labeling chemistry is robust and compatible with HCD analysis on an Orbitrap. Although extreme ratios resulted in higher relative standard deviations, quantification based on an average across multiple peptides nonetheless provided reasonable estimates of relative protein abundance. The latter point suggests that the combination of iTRAQ and Orbitrap HCD will facilitate analysis of biological response, as measured by changes in protein or peptide abundance, across a wide range of conditions.

\section{HCD Provides for Efficient Peptide Identification} of Tryptic Peptides

For higher energy or triple quadrupole like CAD, the collision energy is dependent on both the precursor mass and charge state, following a simple linear equation of the form,

$$
\mathrm{CE}=\text { slope } \times(m / z)+\text { intercept }
$$

where values of the slope and intercept are defined independently for each charge state. The Orbitrap provides for a single, user-defined, normalized collision energy (NCE) for HCD MS/MS experiments. To determine if this variable was appropriately parameterized within the embedded system ITCL code, we acquired HCD MS/MS spectra by LC-MS on equal aliquots of tryptic peptides derived from whole cell lysates (see the Materials and Methods section). Across these acquisitions, we varied the NCE value from $25 \%$ to $50 \%$ in $2.5 \%$ increments. All peptides that exceeded a MASCOT score threshold of 30 (FDR $\sim 0.5 \%$ ) were plotted as a function of NCE (Figure 1a, grey circles). We observed a maximum number of peptide identifications at a NCE $=35$, although the profile was fairly broad, suggesting that good results in toto can be obtained within $\pm 5 \%$ of the optimal NCE. We next re-plotted these data separately for each precursor charge state. In this case we observed that optimal performance exhibited a dependence on precursor charge state, with a maximum number of peptide identifications detected for a NCE of $30 \%$, $37.5 \%$, and $40 \%$, for $2+, 3+$, and $4+$ precursors, respectively.

In addition, we noticed via manual inspection of the data that many seemingly high-quality MS/MS spectra from high charge state $(>2+)$ precursors, went unassigned by MASCOT. We speculated that the fully resolved isotopes associated with each fragment ion contributed sufficient ambiguity to the associated MASCOT searches such that the corresponding spectra were not assigned a peptide sequence with high confidence. To address this question, we created a script to merge peaks within a given isotope cluster, and then charge state-normalized all fragments in the MS/MS spectrum. Using this approach, the number of peptides identified by MASCOT increased $\sim 30 \%$ at $35 \%$ NCE (Figure 1a, grey squares). As expected, the majority of additional identifications were derived from $3+$ and $4+$ precursors (Figure 1a, compare squares and circles for red, blue, and green, respectively). However the relative dependence of peptide identification as a function of normalized collision energy remained unchanged for each charge state.

We next calculated the collision energy (CE) as a function of $\mathrm{m} / \mathrm{z}$ for different charge states (Figure 1b), at a fixed NCE $=35 \%$. This plot confirmed that the default calculation for normalized collision energy followed eq 

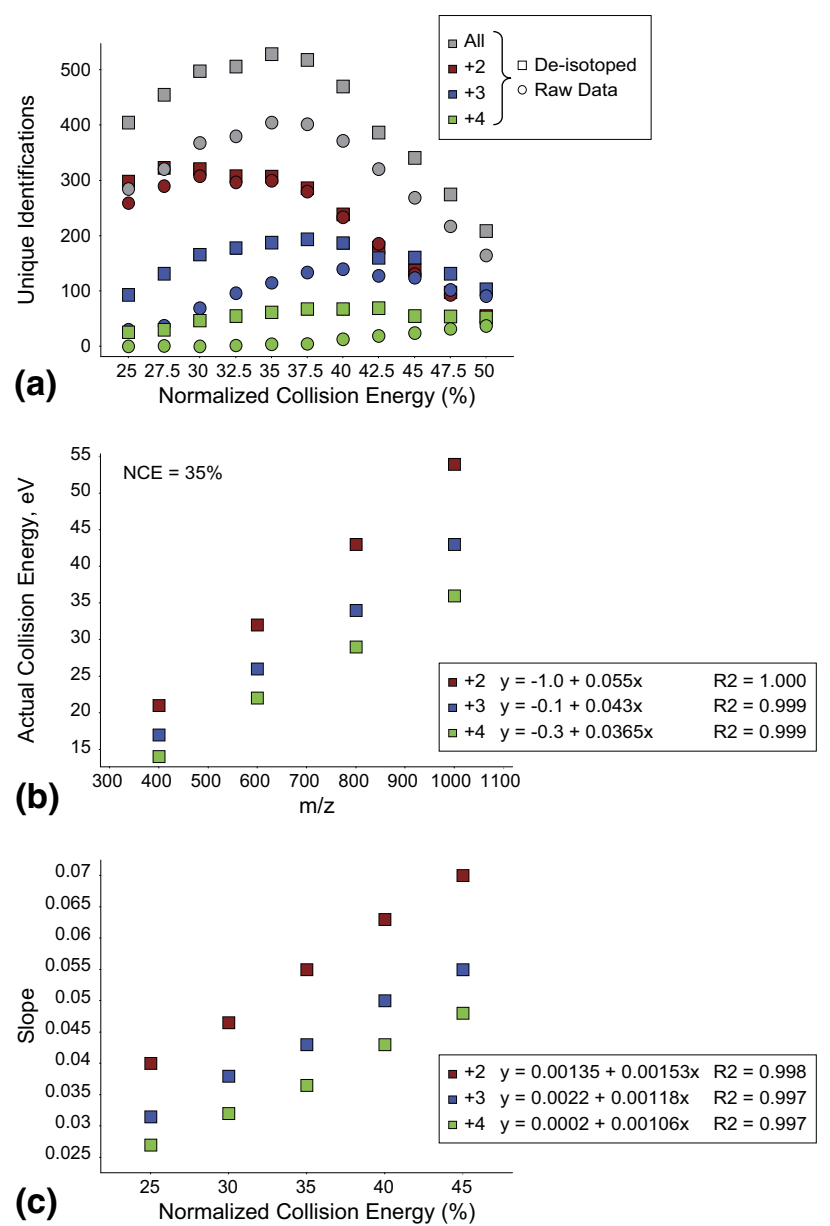

Figure 1. HCD analysis of tryptic peptides from K562 cell lysates. (a) Number of unique peptide identifications (MASCOT Score $>30)$ plotted as a function of HCD normalized collision energy (NCE). Data are plotted for all (gray) and for individual charge states $(2+$, red; $3+$, blue; $4+$, green), and for raw (circles) and de-isotoped (squares) fragment ion spectra. (b) Collision energy $(\mathrm{CE}, \mathrm{eV})$ plotted as a function of precursor $\mathrm{m} / \mathrm{z}$ at $35 \%$ NCE. Precursor charge states of $2+, 3+$, and $4+$ are shown in red, blue, and green, respectively. Data were fit to a linear equation of the form: $C E=$ slope $\times m / z+$ intercept. (c) Slopes for $C E$ versus $m / z$ as in (b), plotted as a function of NCE. Data were fit to a linear equation of the form: slope $=\mathrm{a} \times N C E+\mathrm{b}$.

1. Similarly, a plot of slope versus NCE (Figure 1c) revealed a linear relationship for each charge state examined. A similar analysis (data not shown) demonstrated that the y-intercept varied nonsystematically between 0 and -1 for each charge state examined. These data allowed us to determine the CE for any precursor at any NCE value. Collectively the results in Figure 1 indicate that the higher resolution provided by HCD with Orbitrap detection enables higher confidence MASCOT-based peptide identification through deisotoping of the MS/MS spectra; these data also suggest that independent parameterization of the NCE slope and intercept values as a function of precursor charge state may provide improved results for HCD MS/MS analysis.

\section{HCD Analysis of iTRAQ Labeled Peptides}

We next performed a similar analysis for iTRAQ labeled tryptic peptides derived from whole cell lysate of murine BaF3 cells (Figure 2a). Interestingly, the optimal NCE value shifted somewhat higher for all charge states compared with those observed for unlabeled peptides (Figure 1a). Qualitatively, it also appeared that the number of peptides identified from higher charge states $(>2+)$ was less dependent upon NCE, as evidenced by a broader profile (compare blue and green squares, Figures $1 \mathrm{a}$ and $2 \mathrm{a}$, respectively).

Proteomics quantification based on ITRAQ reagents requires efficient detection of the low-mass reporter ions in addition to the sequence-specific fragments used for peptide identification. To probe iTRAQ-specific performance metrics, we plotted reporter ion intensities as a function of NCE (Figure 2b). These data demonstrated

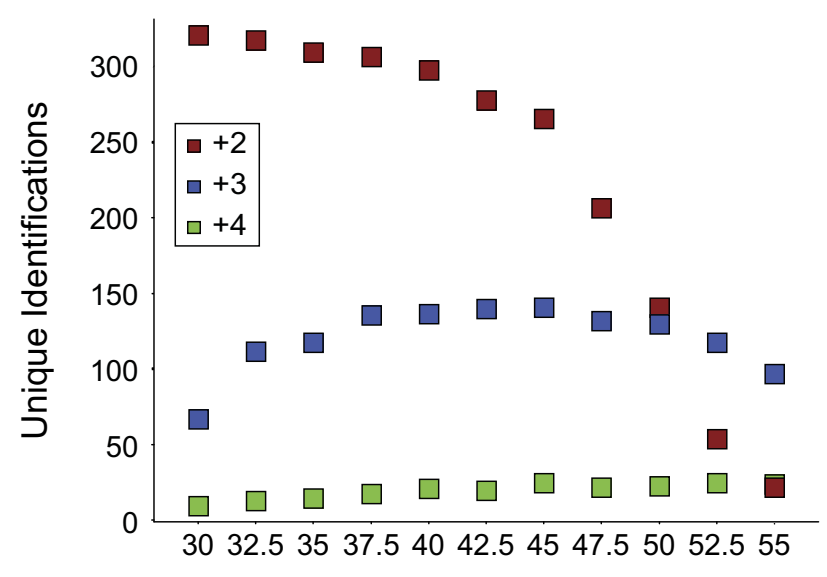

(a)

Normalized Collision Energy (\%)

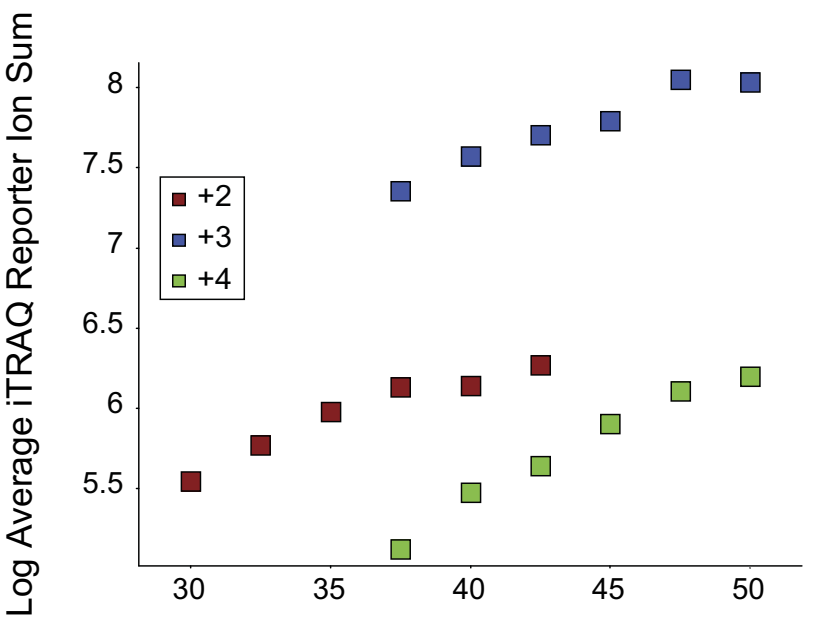

(b)

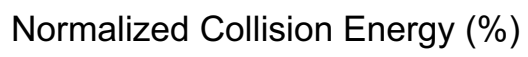

Figure 2. HCD analysis of iTRAQ-labeled tryptic peptides from BaF3 cell lysates. (a) Number of unique peptide identifications (MASCOT Score $>30$ ) plotted as a function of HCD normalized collision energy (NCE). Data are plotted for individual charge states $(2+$, red; $3+$, blue; $4+$, green). (b) Log average of iTRAQ reporter ion intensities for identified peptides plotted as a function of NCE for precursor charge states $2+$ (red), $3+$ (blue), and $4+$ (green). 


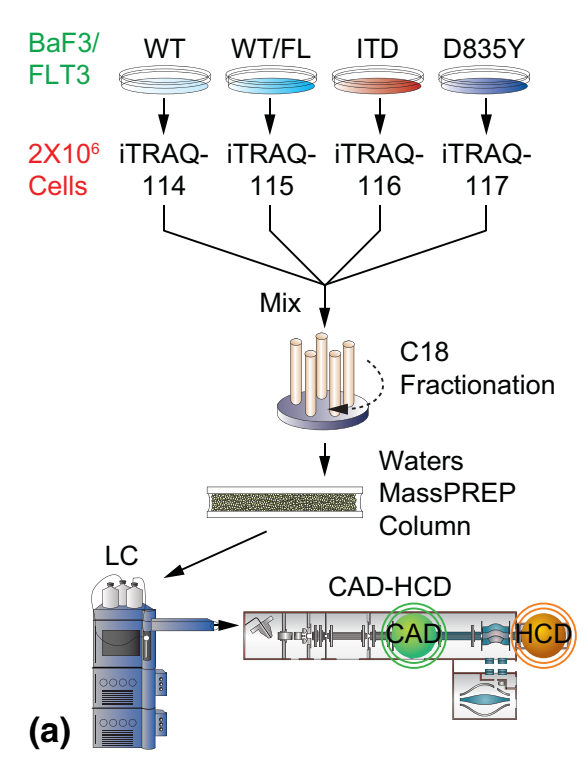

a)

(b)

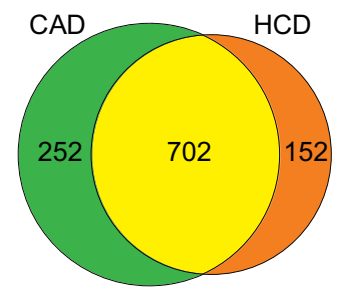

Commonly Identified pSFDYNYR $(594.76,2+)$

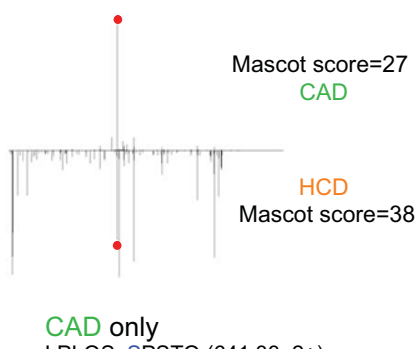

LPLQSpSPSTQ $(641.33,2+)$

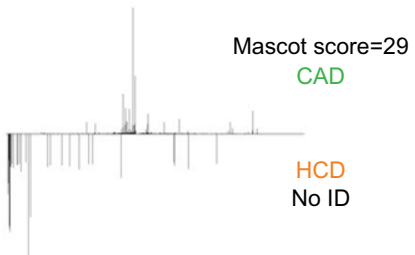

HCD only

YHGHpSMSDPGVpSYR $(632.93,3+)$

(c)

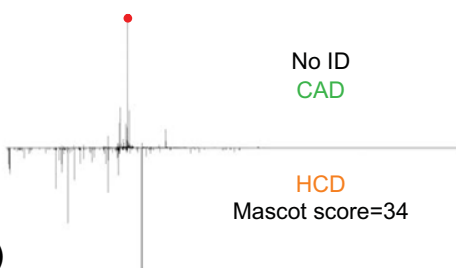

Figure 3. (a) Sample preparation for tissue culture conditions, peptide fractionation, phosphopeptide enrichment, and HCD analysis of AML model system with WT Flt-3 and ITD/D835Y oncogenic Flt-3 mutants. Tryptic phosphopeptides from 2E6 cells were analyzed for each biological condition. Each precursor was subjected to CAD and HCD MS/MS fragmentation on an Orbitrap-XL. (b) Venn diagram for distribution of unique phosphopeptides identified by CAD (green), HCD (orange), or both (yellow). In total, 1106 phosphopeptides were quantified. (c) Representative spectra, displayed as mirror plots with CAD above and HCD below, for phosphopeptides identified with both CAD and HCD (top), CAD alone (middle), and HCD alone (bottom). Red dots illustrate abundant neutral loss of phosphoric acid from precursor (see Supplementary Figure S2 for peak intensity and fragment ion assignments).

that the intensities of iTRAQ reporter ions generally increased as a function of NCE. However comparison of panels $\mathrm{a}$ and $\mathrm{b}$ in Figure 2 reveals that optimization of HCD for iTRAQ labeled peptides requires selection of parameters to provide an acceptable balance between peptide identification and quantification. For example, maximizing reporter ion intensity for precursors of $z>$ 2 may result in a significant decrease in the number of high confidence peptide identifications for doublycharged peptides. Here again, our data suggest that independent adjustment of collision energy dependencies as a function of both precursor $\mathrm{m} / \mathrm{z}$ and charge state may provide improved results for analysis of iTRAQ labeled peptides.

Quantitative Phosphoproteomic Analysis of Flt-3 Signaling in a Model System of Human AML

Our data and results from characterization of HCD on an Orbitrap-XL (Figures 1 and 2) indicated that careful control of collision parameters would likely provide improved performance, especially for quantitative studies that utilize iTRAQ isobaric reagents. As a test, we enriched phosphopeptides from a model system of AML, based on murine BaF3 cells that stably express either WT or constitutively active, oncogenic mutants of the receptor tyrosine kinase Flt-3 (Figure 3a). To increase coverage of the phosphoproteome, iTRAQ-labeled peptides were first subjected to reversed-phase fractionation (see the Materials and Methods section). After further enrichment, phosphopeptides were introduced into an Orbitrap-XL via reversed-phase nanoflow chromatography, and analyzed with a dual scan HCD-CAD acquisition method. In addition, we utilized custom ITCL calls that enabled the embedded system to vary NCE as a function of precursor charge state. Based on our characterization data above and our previous data on sequence analysis of phosphopeptides [19, 23] (and unpublished results), we utilized 40\% NCE 
for doubly-charged precursors and 50\% NCE for triply- and quadruply-charged precursors.

In total, over 1100 unique phosphopeptides on 767 proteins were quantified from five 2-h LC-MS/MS analyses, starting with only $100 \mu \mathrm{g}$ of total protein from each biological condition (WT, WT +FL, ITD, D835Y, Figure 3a). The enrichment efficiency was $>95 \%$, with only 48 nonphosphorylated peptides identified from all five fractions analyzed. Some 702 phosphopeptides were identified by both CAD and HCD, while 252 were uniquely identified by CAD, with the remainder (152 phosphopeptides) uniquely identified by HCD (Figure $3 b$ ). The percentage of singly-, doubly-, and triply- (or higher) phosphorylated peptides was $80 \%, 19 \%$, and $1 \%$, respectively, in good agreement with previous work that correlated large-scale phosphoproteomics data with that resident in the PhophoELM database [19, 23]. Closer inspection of individual spectra revealed that neutral loss of phosphoric acid from the precursor represented a major signal in many of the linear trap CAD scans, but was less pronounced compared with other sequence-specific fragment ions in the HCD spectra (Figure 3c, panel 1). In addition, many of the peptides uniquely identified by CAD had low precursor intensity ( $\leq 5 \mathrm{E} 5$ for the corresponding RIC), relative to all peptides identified in our analysis, and the corresponding HCD scans had insufficient fragment ion current (Figure 3c, panel 2) to support a high-confidence sequence assignment. Nonetheless, a significant number of phosphopeptides were identified only in the HCD scans. Many of these precursors exhibited fragmentation largely restricted to uninformative neutral losses when subjected to MS/MS via CAD in the linear trap (Figure 3c, panel 3).

To further explore the nature of peptides identified by each dissociation method, we next plotted a count of unique phosphopeptide sequences identified as a function of precursor ion intensity (Figure 4a). CAD yielded identification of a higher number of low-intensity $(<5 \mathrm{E} 5$ for the corresponding RIC) phosphopeptides compared with $H C D$, but even for these less abundant species the number of phosphopeptides identified by both methods (yellow bars) was greater than the number identified uniquely by either CAD (green bars) or HCD (orange bars) alone. Interestingly, when we normalized these data (Figure $4 \mathrm{~b}$ ) to the number of phosphopeptides identified by both methods (yellow) or by either $\mathrm{CAD}$ (green) or HCD (orange) alone, we observed very similar distributions. Next we extracted ion injection times for all MS/MS scans and found that the average time for CAD ( $49 \mathrm{~ms}$ ) was significantly less than that for HCD (236 ms). Collectively these data are consistent with recent work from Bantscheff et al. [24], which suggested that Orbitrap-based detection of HCD fragments was less efficient, and corresponded to a longer duty cycle, compared with multiplier-based detection of fragments formed via MS/MS in the linear ion trap.

Despite the results and observations above, the number of phosphopeptides uniquely identified by HCD in our study (Figure $3 \mathrm{~b}$ ) led us to speculate that collision

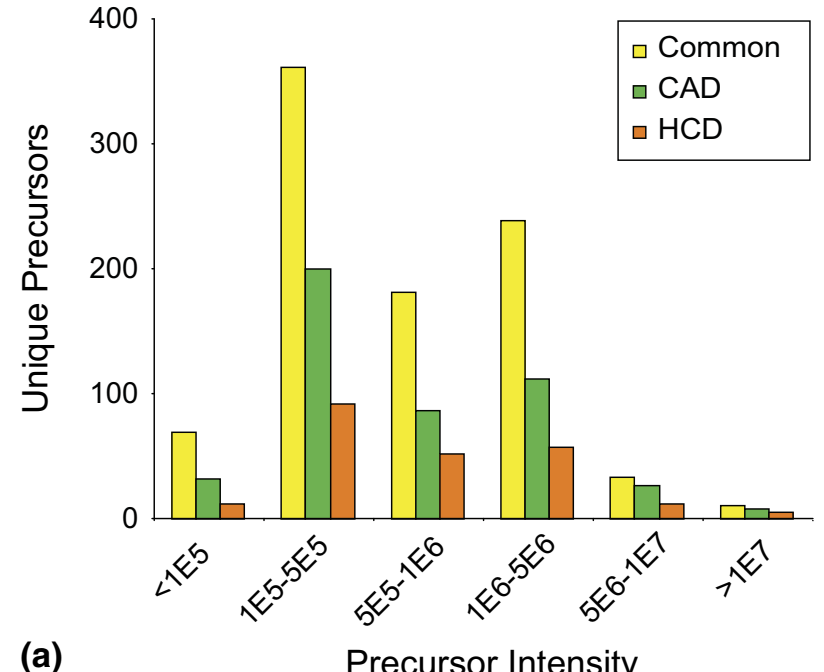

(a) Precursor Intensity

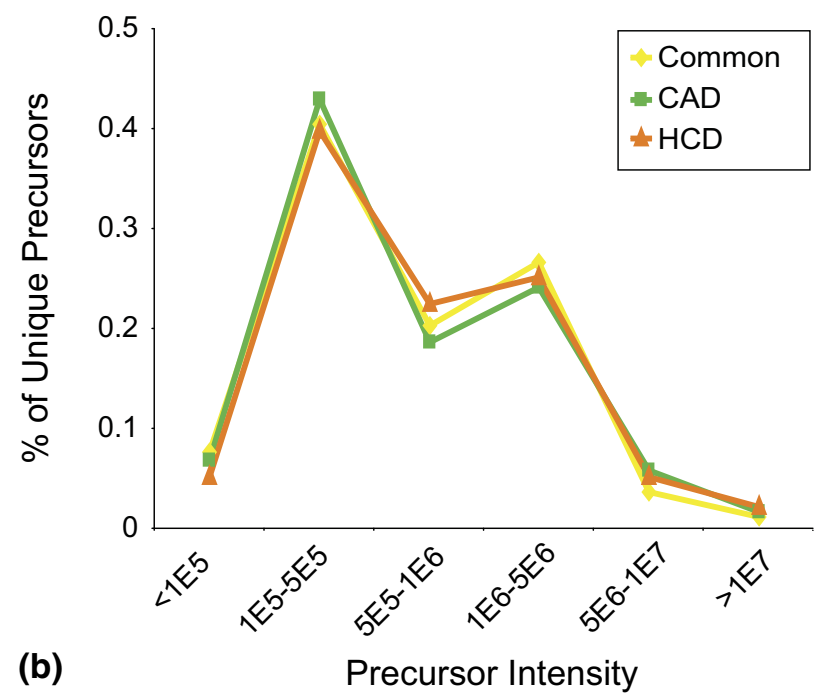

Figure 4. (a) Histogram plot for the number of unique phosphopeptide sequences as a function of binned precursor intensity, for identifications resulting from CAD (green bars), HCD (orange bars), or both methods (yellow bars). (b) Relative percentage of phosphopeptide identifications made via CAD (green), HCD (orange), or both methods (yellow) as a function of binned precursor intensity. The number of peptide identifications in each respective category was used to calculate a relative percent value.

cell-based dissociation may be particularly effective for a sub-class of phosphopeptides, even though the overall duty cycle may suffer as a result of higher ion injection times associated with Orbitrap detection. To evaluate this hypothesis, we plotted the relative percentage of phosphopeptide identifications made via CAD (green), HCD (orange), or both (yellow) as a function of peptide length (Figure 5a). These data revealed a clear bias for identification of short phosphopeptide sequences by collision cell fragmentation relative to identifications that were uniquely associated with CAD. Finally, we plotted the number of unique phosphopeptide sequence identifications as a function of peptide length, and then counted the number of basic amino acids $(\mathrm{R}, \mathrm{K}$, and $\mathrm{H})$ within each bin (Figure 5b-d). Strikingly, these data 


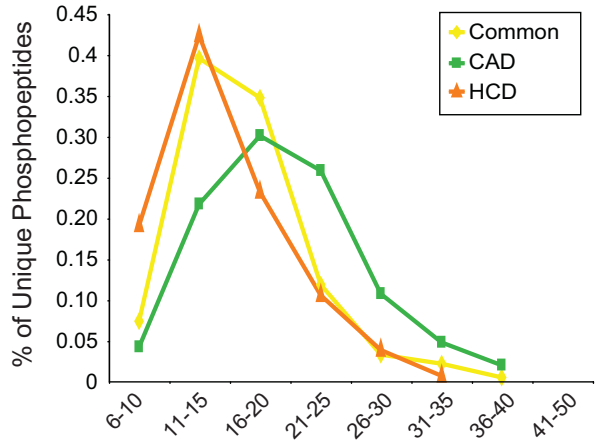

(a)

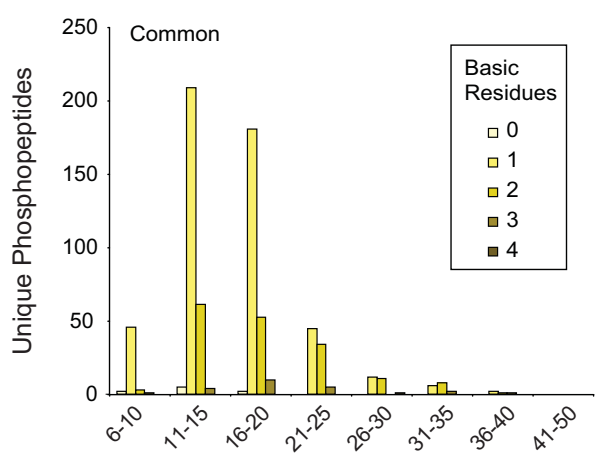

(b)

Peptide Length

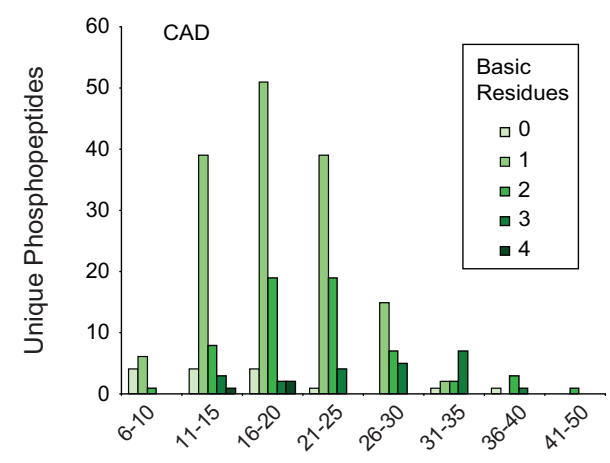

(c)

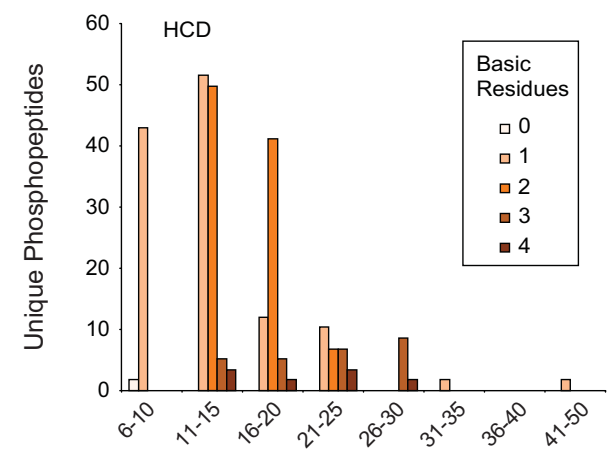

(d)

Peptide Length

Figure 5. Phosphopeptide identification by CAD, $\mathrm{HCD}$, or both methods as a function of peptide length and basicity. (a) Relative percentage of phosphopeptide identifications made via CAD (green), HCD (orange), or both methods (yellow) plotted as a function of binned peptide length. (b-d) Histogram plots for the number of unique phosphopeptide sequences as a function of peptide length. The number of basic amino acids ( $R, K$, and $H$ ) is indicated in each peptide length bin for identifications made by both methods (b), or observed uniquely with CAD (c), or HCD (d) fragmentation.

revealed that short phosphopeptides with internal basic residues are particularly amendable to sequence analysis by HCD. We suspect that ion-neutral collisions in the HCD cell are sufficiently energetic such that amide bond cleavage effectively competes with neutral loss of phosphoric acid, leading to improved sequence coverage for this class of peptides compared with low-energy $\mathrm{CAD}$ in the linear ion trap.

The use of HCD-based MS/MS in our analysis provided for quantification of 702 phosphorylation sites downstream of WT and mutant FLT-3 kinases. This system is a model of oncogenic signaling in acute myeloid leukemia. Both the internal tandem duplication (ITD) and point mutant (D835Y) kinases are transformative, although they carry different clinical prognoses, and exhibit varying responses to the current suite of targeted, small molecule FLT-3 inhibitors [25]. These observations suggest that the mutants may activate different downstream targets. To explore this scenario in our quantitative proteomics data, we plotted individual phosphopeptides based on the corresponding iTRAQ reporter ion ratios for each mutant normalized to signaling present in the context of the WT receptor (Figure 6). We used a global significance threshold of \pm 1 .5-fold to further subdivide the data into eight quadrants. This approach provided a general framework for assignment of specific phosphorylation sites as up- or downregulated, and as associated with signaling downstream of one or both mutant kinases. Supplementary Table 1 provides peptide sequence and related data from Figure 6. We next used a Fisher's exact test to identify enrichment of KEGG pathways [26] based on proteins derived from phosphorylated peptides in each quadrant.

Both D835Y and ITD mutants support factorindependent growth of cells. As expected, the data in quadrant no. 1 show that signaling from both mutants coordinately activates proliferative and survival pathways, most notably members in the insulin, phosphatidylinositol, and ErbB signaling pathways. Peptides from a few specific proteins (Pkm2, Pi3k, Cam, Shc1, and Erk2) in these pathways are highlighted in Figure 6. Inspection of quadrant eight reveals that the point mutant preferentially activates proteins involved in immune system signaling. For example, Tec and Shp1 are both critical mediators of normal hematopoiesis [27, 28] and, when dysregulated, may contribute to leukemogenesis [29, 30]. These and other proteins listed in 


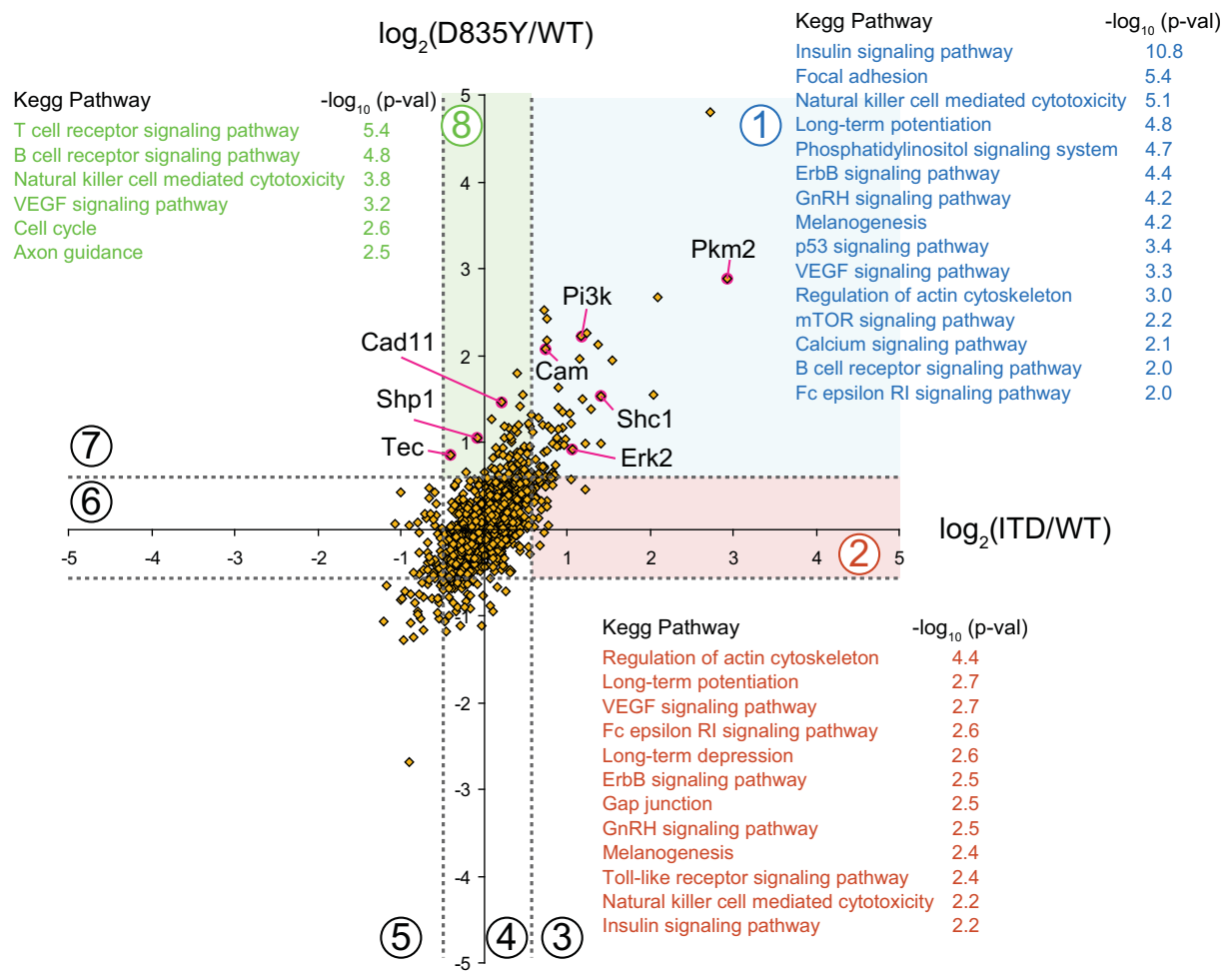

Figure 6. Scatter plot for measured ratios of ITD (x-axis) and D835Y (y-axis) normalized to WT Flt-3 (see also Supplement Table 1). Dashed lines represent global thresholds based on a \pm 1.5 -fold change between each mutant and WT Flt-3, respectively. Proteins corresponding to phosphopeptides in each quadrant were further analyzed for enrichment of Kegg Pathways (see Methods). Specific pathways with $P \leq 0.01$ are listed for quadrants associated with proteins activated by D835Y (green), ITD (red), or both (blue).

Supplementary Table 1 may represent key mediators that drive transformation of the leukemic phenotype.

\section{Conclusions}

Collectively, our results demonstrate that HCD on the Orbitrap-XL platform performs well for sequence analysis of both tryptic peptides and their iTRAQ labeled counterparts. In addition, our data suggest that independent control of the collision energy parameters as a function of precursor charge state may provide more uniform performance in terms of peptide sequence identification across multiple charge states. Similar to recent work by Bantscheff et al. [24] for PQD, our results demonstrate the nuanced nature of peptide identification versus quantification, and suggest that so-called hybrid instrument geometries, while powerful and flexible, can also benefit from continued refinement of acquisition parameters. We utilized a combined CADHCD scan mode in conjunction with iTRAQ labeling for quantitative analysis of signaling cascades in a model system of human myeloid leukemia. Consistent with previous studies [24], we observed that CAD yielded more high-confidence phosphopeptide sequence identifications compared with HCD. Unexpectedly, our results demonstrated that HCD-based MS/MS performs particularly well for identification of small phos- phopeptides carrying internal basic residues. When analyzed in the context of biological pathways, our quantitative phosphoproteomics data suggest potential points of divergent signaling downstream of clinically relevant FLT-3 kinase mutants; further detailed biochemical studies directed at these proteins and pathways may reveal valuable insight into the molecular mechanisms of transformation associated with oncogenic FLT-3 activity in AML.

\section{Acknowledgments}

The authors thank Dr. Michael Senko at ThermoFisher Scientific for providing ITCL code and guidance in customization of HCD collision energy parameters. The authors also thank Eric Smith for helpful discussion and preparation of the figures, and Manor Askenazi for assistance with data analysis. The authors acknowledge support for this work by the Dana-Farber Cancer Institute.

\section{Appendix A Supplementary Material}

Supplementary material associated with this article may be found in the online version at doi:10.1016/ j.jasms.2009.03.019. 


\section{References}

1. Hunt, D. F.; Buko, A. M.; Ballard, J. M.; Shabanowitz, J.; Giordani, A. B. Sequence Analysis of Polypeptides by Collision Activated Dissociation on a Triple Quadrupole Mass Spectrometer. Biomed. Mass Spectrom. 1981, 8, 397-408.

2. Schwartz, J. C.; Syka, J. E. P.; Jardine, I. High Resolution on a Quadrupole Ion Trap Mass Spectrometer. J. Am. Soc. Mass Spectrom 1991, 2, 198-204

3. Stafford, G. C.; Kelley, P. E.; Syka, J. E. P.; Reynolds, W. E.; Todd, J. F. J. Recent Improvements in and Analytical Applications of Advanced Ion Trap Technology. Int. J. Mass Spectrom. Ion Processes 1984, 60, 85-98.

4. Louris, J. N.; Amy, J. W.; Ridley, T. Y.; Cooks, R. G. Injection of Ions into a Quadrupole Ion Trap Mass Spectrometer. Int. J. Mass Spectrom. Ion Processes 1989, 88, 97-111.

5. McLuckey, S. A.; Van Berkel, G. J.; Glish, G. L.; Huang, E. C.; Henion, J. D. Ion Spray Liquid Chromatography/Ion Trap Mass Spectrometry Determination of Biomolecules. Anal. Chem. 1991, 63, 375-383.

6. Van Berkel, G. I.; Glish, G. L.; McLuckey, S. A. Electrospray Ionization Combined with Ion Trap Mass Spectrometry. Anal. Chem. 1990, 62, 1284-1295.

7. Kaiser, R. E.; Louris, J. N.; Amy, J. W.; Cooks, R. G. Extending the Mass Range of the Quadrupole Ion Trap Using Axial Modulation. Rapid Commun. Mass Spectrom. 1989, 3, 225-229.

8. Stafford, G. C.; Taylor, D. M.; Bradshaw, S. C.; Syka, J. E. P.; Uhrich, M Enhanced Sensitivity and Dynamic Range on an Ion Trap Mass Spectrometer with Automatic Gain Control. Proceedings of the 35th Annual Conference on Mass Spectrometry and Allied Topics; Denver, CO, 1987.

9. Wilkins, M. R.; Sanchez, J. C.; Gooley, A. A.; Appel, R. D.; HumpherySmith, I.; Hochstrasser, D. F.; Williams, K. L. Progress with Proteome Projects: Why All Proteins Expressed by a Genome Should be Identified and How to Do It. Biotechnol. Genetic Eng. Rev. 1995, 13, 19-50.

10. Morris, H. R.; Paxton, T.; Dell, A.; Langhorne, J.; Berg, M.; Bordoli, R. S.; Hoyes, J.; Bateman, R. H. High Sensitivity Collisionally-Activated Decomposition Tandem Mass Spectrometry on a Novel Quadrupole/ Orthogonal-Acceleration Time-of-Flight Mass Spectrometer. Rapid Commun. Mass Spectrom. 1996, 10, 889-896.

11. Makarov, A. Electrostatic Axially Harmonic Orbital Trapping: A HighPerformance Technique of Mass Analysis. Anal. Chem. 2000, 72, 11561162.

12. Syka, J. E.; Marto, J. A.; Bai, D. L.; Horning, S.; Senko, M. W.; Schwartz, J. C.; Ueberheide, B.; Garcia, B.; Busby, S.; Muratore, T.; Shabanowitz, J.; Hunt, D. F. Novel Linear Quadrupole Ion Trap/FT Mass Spectrometer: Performance Characterization and Use in the Comparative Analysis of Histone H3 Post-Translational Modifications. I. Proteome Res. 2004, 3 , $621-626$.

13. Schwartz, J. C.; Senko, M. W.; Syka, J. E. A Two-Dimensional Quadrupole Ion Trap Mass Spectrometer. I Am. Soc. Mass Spectrom. 2002, 13 659-669.

14. Hager, J. W.; Yves Le Blanc, C. Product Ion Scanning Using a Q-Q-Q Linear Ion Trap (Q Trap) Mass Spectrometer. Rapid Commun. Mass Spectrom. 2003, 17, 1056-1064.
15. Mann, M.; Ong, S.-E.; Grønborg, M.; Steen, H.; Jensen, O. N.; Pandey, A. Analysis of Protein Phosphorylation Using Mass Spectrometry: Deciphering the Phosphoproteome. Trends Biotechnol. 2002, 20, 261-268.

16. Khidekel, N.; Ficarro, S. B.; Peters, E. C.; Hsieh-Wilson, L. C. Exploring the O-GlcNAc Proteome: Direct Identification of O-GlcNAc-Modified Proteins from the Brain. Natl. Acad. Sci. U.S.A. 2004, 101, 13132-13137.

17. Moyer, S. C.; Cotter, R. J.; Woods, A. S. Fragmentation of Phosphopeptides by Atmospheric Pressure MALDI and ESI/Ion Trap Mass Spectrometry. J. Am. Soc. Mass Spectrom. 2002, 13, 274-283.

18. Palumbo, A. M.; Tepe, J. J.; Reid, G. E. Mechanistic Insights into the Multistage Gas-Phase Fragmentation Behavior of Phosphoserine- and Phosphothreonine-Containing Peptides. J. Proteome Res. 2008, 7, 771779 .

19. Ndassa, Y. M.; Orsi, C.; Marto, J. A.; Chen, S.; Ross, M. M. Improved Immobilized Metal Affinity Chromatography for Large-Scale Phosphoproteomics Applications. I. Proteome Res. 2006, 5, 2789-2799.

20. Moser, K.; White, F. M. Phosphoproteomic Analysis of Rat Liver by High Capacity IMAC and LC-MS/MS. J. Proteome Res. 2006, 5, 98-104.

21. Olsen, J. V.; Macek, B.; Lange, O.; Makarov, A.; Horning, S.; Mann, M. Higher-Energy C-Trap Dissociation for Peptide Modification Analysis. Nat. Methods 2007, 4, 709-712.

22. Ross, P. L.; Huang, Y. N.; Marchese, J. N.; Williamson, B.; Parker, K.; Hattan, S.; Khainovski, N.; Pillai, S.; Dey, S.; Daniels, S.; Purkayastha, S.; Juhasz, P.; Martin, S.; Bartlet-Jones, M.; He, F.; Jacobson, A.; Pappin, D. J. Multiplexed Protein Quantitation in Saccharomyces cerevisiae Using Amine-Reactive Isobaric Tagging Reagents. Mol. Cell. Proteom. 2004, 3 , $1154-1169$.

23. Ficarro, S. B.; Parikh, J. R.; Blank, N. C.; Marto, J. A. Niobium(V) Oxide (Nb205): Application to Phosphoproteomics. Anal. Chem. 2008, 80, 4606-4613

24. Bantscheff, M.; Boesche, M.; Eberhard, D.; Matthieson, T.; Sweetman, G.; Kuster, B. Robust and Sensitive ITRAQ Quantification on an LTQ Orbitrap Mass Spectrometer. Mol. Cell. Proteom. 2008, 7, 1702-1713.

25. Knapper, S. Flt3 Inhibition in Acute Myeloid Leukaemia. Br. J Haematol. 2007, 138, 687-699.

26. Kanehisa, M. A Database for Post-Genome Analysis. Trends Genet. 1997, 13, 375-376.

27. Berg, L. J. Signaling through Tec Kinases Regulates Conventional Versus Innate Cd8(+) T-Cell Development. Nat. Rev. Immunol. 2007, 7, 479-485.

28. Paling, N. R.; Welham, M. J. Tyrosine Phosphatase Shp-1 Acts at Different Stages of Development to Regulate Hematopoiesis. Blood 2005, 105, 4290-4297.

29. Bittorf, T.; Seiler, J.; Zhang, Z.; Jaster, R.; Brock, J. Shp1 Protein Tyrosine Phosphatase Negatively Modulates Erythroid Differentiation and Suppression of Apoptosis in J2e Erythroleukemic Cells. Biol. Chem. 1999, 380, 1201-1209.

30. Feldhahn, N.; Klein, F.; Mooster, J. L.; Hadweh, P.; Sprangers, M. Wartenberg, M.; Bekhite, M. M.; Hofmann, W. K.; Herzog, S.; Jumaa, H. Rowley, J. D.; Muschen, M. Mimicry of a Constitutively Active Pre-B Cell Receptor in Acute Lymphoblastic Leukemia Cells. J. Exp. Med. 2005, 201, 1837-1852. 